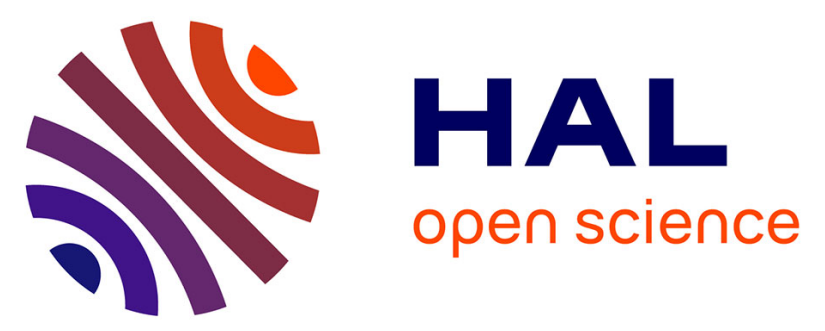

\title{
Effect of the sol fraction and hydrostatic deformation on the viscoelastic behavior of prestrained highly filled elastomers
}

Aurélie Azoug, Andrei Constantinescu, Rachel Marie Praidelles-Duval, M. F. Vallat, R. Nevière, Bassel Haidar

\section{To cite this version:}

Aurélie Azoug, Andrei Constantinescu, Rachel Marie Praidelles-Duval, M. F. Vallat, R. Nevière, et al.. Effect of the sol fraction and hydrostatic deformation on the viscoelastic behavior of prestrained highly filled elastomers. Journal of Applied Polymer Science, 2012, 127 (3), pp.1772-1780. 10.1002/app.37800 . hal-00761141

HAL Id: hal-00761141

https://hal-polytechnique.archives-ouvertes.fr/hal-00761141

Submitted on 25 Sep 2020

HAL is a multi-disciplinary open access archive for the deposit and dissemination of scientific research documents, whether they are published or not. The documents may come from teaching and research institutions in France or abroad, or from public or private research centers.
L'archive ouverte pluridisciplinaire HAL, est destinée au dépôt et à la diffusion de documents scientifiques de niveau recherche, publiés ou non, émanant des établissements d'enseignement et de recherche français ou étrangers, des laboratoires publics ou privés.

\section{(ㄷ)(i)}

Distributed under a Creative Commons Attribution| 4.0 International License 


\title{
Effect of the Sol Fraction and Hydrostatic Deformation on the Viscoelastic Behavior of Prestrained Highly Filled Elastomers
}

\author{
A. Azoug, ${ }^{1}$ A. Constantinescu, ${ }^{1}$ R. M. Pradeilles-Duval, ${ }^{1}$ M. F. Vallat, ${ }^{2}$ R. Nevière, ${ }^{3}$ B. Haidar ${ }^{2}$ \\ ${ }^{1}$ Laboratoire de Mécanique des Solides-CNRS UMR 7649, Ecole Polytechnique, 91128 Palaiseau Cedex, France \\ ${ }^{2}$ Institut de Science des Matériaux de Mulhouse-LRC 7228 CNRS-UHA, 15 Rue Jean Starcky-BP 2488, 68057 Mulhouse \\ Cedex, France \\ ${ }^{3}$ SNPE Matériaux Energétiques, Centre de Recherches du Bouchet, 9 Rue Lavoisier, 91710 Vert-le-Petit, France
}

Correspondence to: A. Azoug (E-mail: aurelie.azoug@polytechnique.edu)

\begin{abstract}
This study focuses on the relations between the microstructure and the viscoelastic behavior of an industrial solid propellant belonging to the class of highly filled elastomers. Precisely, the study aims at determining the impact on the viscoelastic behavior of the presence of the sol fraction inside the polymer network. The sol fraction is the part of the binder that a good solvent can extract. The solid propellant is swollen to various extents by solutions of plasticizer and polymer molecules. This swelling leads to a hydrostatic deformation of the polymer network, corresponding to an extension or contraction loading for each specimen. Prestrained dynamic mechanical analysis tests, superimposing a small oscillating strain on a prestrain, characterize the viscoelastic behavior. The degree of swelling of the network and the effective filler fraction drive the viscoelastic response. In addition, the mechanical behavior does not depend on the chemical nature of the introduced sol fraction. Moreover, a nonlinear behavior, i.e., an increase in both storage and loss moduli with increasing prestrain, is initiated at low prestrain. This nonlinearity depends on the contraction or extension of the network and could result from particles aligning with prestrain, which is expected in such highly filled materials. (C) 2012 Wiley Periodicals, Inc. J. Appl. Polym. Sci. 127: 1772-1780, 2013
\end{abstract}

KEYWORDS: elastomers; fillers; swelling; propellant; reinforcement; viscoelastic properties; structure-property relations

\section{INTRODUCTION}

Solid propellants are highly filled elastomers used for propulsion of rockets and launchers. Highly filled elastomers have a filler volume fraction of up to $80 \%$. The incorporation of such a high quantity of fillers has two main consequences. On one hand, only a small amount of elastomeric binder holds the particles together, although the viscoelastic behavior originates from this polymeric binder. However, adding fillers to a polymer fundamentally modifies its viscoelastic properties. Consequently, these highly filled materials exhibit a complex nonlinear viscoelastic mechanical behavior, which is rather problematic to model. The offered models either are phenomenological ${ }^{1-3}$ or go beyond classical homogenization theories. ${ }^{4-8}$ Because of complex binder-filler and filler-filler interactions on multiple length scales, local deformation mechanisms are not clearly determined.

The main challenge in manufacturing solid propellants remains incorporating a high quantity of fillers. Moreover, the final product must exhibit the targeted mechanical properties, princi- pally low viscoelastic dissipations and high elongation at break, despite the low volume fraction of binder. To reach both these goals, chemists use a carefully optimized combination of the plasticizing and cross-linking effects. First, a high quantity of plasticizer molecules is added to the binder before curing it. These molecules not only facilitate the process but also reduce the viscous dissipations because of intermolecular frictions in the final material. Second, the cross-linking of the polymer network is intentionally incomplete. Consequently, some polymer chains remain unlinked to the network and thus are extractable. This low cross-link density increases the elongation at break of the binder, despite the high quantity of fillers. The plasticizers partially counteract the consequent increase in viscous dissipations.

In solid propellants, unlinked polymer chains and plasticizer molecules constitute the phase of the microstructure named sol fraction. The sol fraction is defined as the phase that a good solvent can extract and, therefore, that does not contribute to the elastic response. Only few studies have investigated the 
Table I. Composition of Tested Specimens

\begin{tabular}{|c|c|c|c|c|c|c|}
\hline & \multirow[b]{2}{*}{ Fillers (\%) } & \multirow[b]{2}{*}{ Swelling (\%) } & \multicolumn{2}{|c|}{ Sol fraction } & \multirow{2}{*}{$\begin{array}{l}\text { Plasticizer } \\
\% \text { of binder }\end{array}$} & \multirow[b]{2}{*}{$T_{g}\left({ }^{\circ} \mathrm{C}\right)$} \\
\hline & & & Composition & $\%$ of binder & & \\
\hline I & 80.0 & 36.3 & HTPB DOZ & 67.5 & 26.6 & -83.6 \\
\hline$E$ & 92.5 & -55.7 & - & 0.0 & 0.0 & - \\
\hline$P$ & 87.5 & -22.1 & HTPB & 43.1 & 0.0 & -74.1 \\
\hline$P_{\text {oxy }}$ & 88.3 & -27.9 & Cross-linked HTPB & 38.6 & 0.0 & -75.5 \\
\hline DP & 87.7 & -23.1 & HTPB/DOZa & 42.4 & $32.6^{a}$ & -85.1 \\
\hline D & 56.9 & 313.8 & DOZ & 89.3 & 89.3 & -103.4 \\
\hline
\end{tabular}

aSee the Results and Discussion section: glass transition temperature analysis.

influence of the presence of this sol fraction on the mechanical viscoelastic behavior of network elastomers ${ }^{9,10}$ and filled elastomers. ${ }^{11}$ Essentially, the impact of the sol fraction is still uncertain and has not been investigated in highly filled elastomers. Thus this work aims at specifying the influence of the sol fraction, in nature and quantity, on the viscoelastic properties.

Manufacturing propellants at various levels of cross-linking would modify both the sol fraction and the polymer network, because increasing one is equivalent to decreasing the other. Moreover, the plasticizer interferes with the chemical cross-linking reaction and modifies the resulting network. ${ }^{12}$ Consequently, adding plasticizer molecules before curing has an influence on the structure of the obtained network. In this study, plasticizers are added after curing and we compare identical networks swollen with variable amounts of soluble polymer and plasticizer. The chosen method constitutes a more rigorous procedure to show the effect of sol fraction but does not amount to the industrial manufacturing process.

The prestrained dynamic mechanical analysis (PDMA) superimposes small strain oscillations on a tensile prestrain. This technique measures the highly nonlinear viscoelastic behavior of elastomers under complex loading conditions. This procedure has been previously discussed on unfilled rubber in tensile and torsion modes, ${ }^{10,13-15}$ on carbon black- or silica-filled elastomers in tensile and shear modes, ${ }^{11,15-21}$ and on highly filled elastomers in torsion mode. ${ }^{22}$ In each case, adding a prestrain to the loading strongly modifies the measured nonlinear behavior.

Finally, the response to PDMA loading has relevance to industrial applications. During the manufacturing of a rocket motor, the polymer is mixed with fillers, heated during curing, and then cooled. As a result, the material exhibit residual stresses, which constitute a prestrain to any further loading. At initiation, the motor experiences a high frequency oscillatory loading that can lead to failure of the material. The safety of propulsion devices depends directly on the accurate modeling of the response under superimposed loading.

The article is organized as follows. The tested materials are presented. The experimental procedures are described and a mathematical model for the viscoelastic behavior measured by PDMA tests is introduced. Finally, the results of swelling experiments, differential scanning calorimetry (DSC), and PDMA are reported and discussed.

\section{Materials}

The solid propellant studied here is a highly filled elastomer characterized by a filler volume fraction of $80 \%$. The fillers are ammonium perchlorate and aluminum particles. The binder is based on hydroxy-terminated polybutadiene (HTPB) prepolymer (the functionality of the polymer is slightly higher than 2) cured with a methylene dicyclohexyl isocyanate (MDCI). The plasticizer introduced in the mixture is dioctyl azelate (DOZ) molecules. The material is thermally cured for 2 weeks at $50^{\circ} \mathrm{C}$.

The $\mathrm{NCO} / \mathrm{OH}$ ratio is the ratio of the molar quantities of MDCI and HTPB prepolymer introduced during manufacturing. Because here $\mathrm{NCO} / \mathrm{OH}$ ratio is 0.8 , the system does not contain enough crosslinking agents to create a complete network. As a consequence, part of the HTPB molecules remains unlinked to the network. The sol fraction contains both the unlinked HTPB chains and the plasticizer molecules.

Several specimens are prepared, first, by extraction to a constant mass of this reference propellant by a solvent and, second, by the absorption of the different constituents as listed in Table I. Therefore, the sol fraction in these specimens is modified whereas the polymer network and the fillers stay unaltered. The experimental section details the preparation procedure.

\section{EXPERIMENTAL}

In this work, specimens are designed using extraction and absorption experiments to control the composition of the sol fraction. DSC tests measure the glass transition temperature of each specimen. PDMA allows the comparison between mechanical behaviors.

\section{Material Preparation by Swelling}

New specimens are manufactured in two steps: (i) extraction of the sol fraction to constant mass by swelling in a good solvent and (ii) absorption of a modified sol fraction by swelling with different constituents (Figure 1).

(i) Extraction: The propellant I is immersed in toluene until a constant mass is reached, in this case during 1 week, and the solvent is renewed every $24 \mathrm{~h}$. As the solvent swells the material, free molecules are diffused out of the system. The extraction of the sol fraction of specimen I produces specimen E. Mass loss $M_{\mathrm{sf}}$ after extraction and drying indicates the mass of sol fraction in the initial 


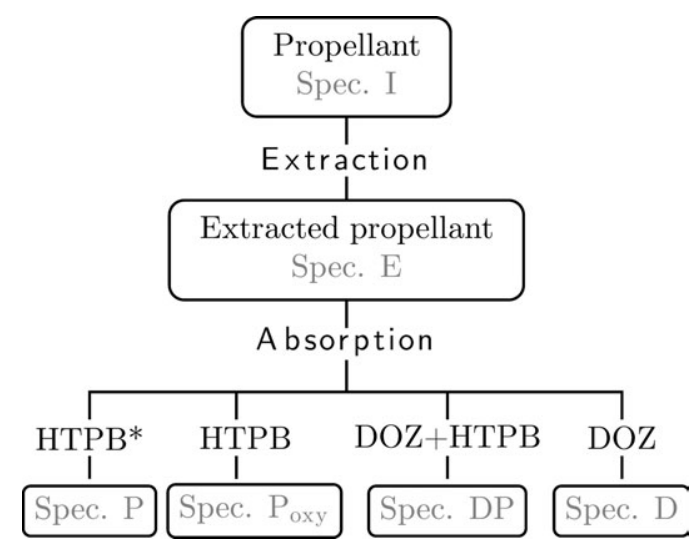

Figure 1. Design of propellant specimens by swelling. Spec., specimen; $\mathrm{HTPB}^{*}$, HTPB with antioxidizer.

formulation. The plasticizer molecules are introduced into the reference propellant before curing. Desgardin et al. ${ }^{23}$ performed the extraction of a similar propellant with the same procedure as the one described in the previous paragraph. The quantity of plasticizer extracted was measured and was equal to the quantity introduced during manufacturing. This result proves that, as the polymer is cured, no link is formed between the plasticizer and the polymer network or the fillers. Thus, the mass of plasticizer $M_{\text {plast }}$ in the sol fraction of specimen I is known and corresponds to the mass of plasticizers introduced during the manufacturing of the material. The mass of polymer chains in the sol fraction $M_{\text {pol.sol. }}$ is then deduced using eq. (1),

$$
M_{\text {pol.sol. }}=M_{\text {sf }}-M_{\text {plast }} .
$$

Finally, the volume of the measured sol fraction $V_{\mathrm{sf}}$ is deduced according to eq. (2),

$$
V_{\text {sf }}=\frac{M_{\text {pol.sol. }}}{\rho_{\text {pol.sol. }}}+\frac{M_{\text {plast }}}{\rho_{\text {plast }}},
$$

where $\rho_{\text {pol.sol. }}$ and $\rho_{\text {plast }}$ are the densities of the polymer in the sol fraction and of the plasticizer, $910 \mathrm{~kg} / \mathrm{m}^{3}$ and $920 \mathrm{~kg} /$ $\mathrm{m}^{3}$, respectively.

(ii) Absorption: The extracted specimen is then swollen with different constituents. For instance, by immersing specimen $\mathrm{E}$ in pure HTPB prepolymer (with antioxidizer to prevent microgel formation), specimen $\mathrm{P}$ is obtained. Specimen $\mathrm{P}_{\text {oxy }}$ is obtained by a similar procedure using a pure HTPB prepolymer bath. The immersing bath is heated to $60^{\circ} \mathrm{C}$ during 1 month to enhance polymer chain mobility and to enable HTPB penetration into the specimen. It is noticed that for the specimen $\mathrm{P}_{\text {oxy }}$, heating also causes oxidation and microgel formation in the substituted soluble polymer.

Material E is immersed in DOZ (plasticizer) to obtain specimen $\mathrm{D}$ or in a 70/30 blend of HTPB prepolymer and DOZ for specimen DP. Because of the presence of the plasticizer, these specimens do not need to be heated to generate product penetration.
All samples are then dried to constant mass. The increase in volume after this step is calculated from the mass gain and the densities of polymer and plasticizer.

The degree of swelling of the network is different in each specimen according to this volume increase. The volume of the binder without any plasticizer is considered as the reference state, $V_{\text {ref. }}$ In this reference state, the binder contains only polymer chains, crosslinked or not, which are therefore at their unperturbed state.

The volume of the binder $V_{\text {binder }}$ is calculated after absorption from eq. (3). The swelling is then quantified by the relative volume difference between the final and reference states [eq. (4)]. Finally, the volume filler fraction is determined.

$$
\begin{aligned}
& V_{\text {binder }}=V_{\text {specimen }}-V_{\text {fillers }}, \\
& \text { Swelling }=\frac{V_{\text {binder }}-V_{\text {ref }}}{V_{\text {ref }}} .
\end{aligned}
$$

\section{DSC}

The glass transition temperature $T_{g}$ is determined by a DSC measurement, performed using a Mettler Toledo DSC 30 apparatus. Measurements of $T_{g}$ take place in nitrogen atmosphere, at a flow rate of $40 \mathrm{ml} / \mathrm{min}$. The specimens undergo two consecutive temperature cycles, cooling from $20^{\circ} \mathrm{C}$ to $-120^{\circ} \mathrm{C}$ at a cooling rate of $-20^{\circ} \mathrm{C} / \mathrm{min}$, and heating from $-120^{\circ} \mathrm{C}$ to $100^{\circ} \mathrm{C}$ at a heating rate of $5^{\circ} \mathrm{C} / \mathrm{min}$. The temperature, $T_{g}$, and the specific heat change at $T_{g}, \Delta C_{p}$, are measured in the heating phase of each cycle and the determined $T_{g}$ value is the mean value of the inflexion points during each cycle.

The specific heat change $\Delta C_{p}$ is a measure of the change in segmental mobility as the material undergoes the glass transition. Because the measured glass transition involves only the organic phase, the value of $\Delta C_{p}$ depends on the binder fraction of the material, which, in turn, depends on the swelling degree reached in the absorption step of the material preparation. To compare the specimens, the specific heat change $\Delta C_{p}$ is normalized with respect to the binder fraction in weight percentage. The normalized specific heat change is expressed as Joules per gram of binder per degree Celsius.

\section{PDMA}

PDMA experiments are performed using a Metravib Viscoanalyseur VA3000. Dumbbell specimens of length $50 \mathrm{~mm}$ and rectangular section $10 \times 5 \mathrm{~mm}^{2}$ are used. The PDMA procedure consists in applying simultaneously to the specimen a tensile prestrain and a sinusoidal strain. The total applied strain according to the time $t$ is

$$
\varepsilon(t)=\varepsilon_{0}+\varepsilon_{a} \sin (\omega t)
$$

where the single strain amplitude is $\varepsilon_{a}=0.01 \%$ and the pulsation $\omega=2 \pi f$. The frequency is $f=5 \mathrm{~Hz}$. The tests are performed at room temperature. Different levels of prestrain $\varepsilon_{0, i}$ are reached (Figure 2) from $0.01 \%$ to about $10 \%$.

The norm of the complex modulus $E^{\star}$ and the loss factor $\tan \delta$ are measured. The storage and loss moduli, $E^{\prime}$ and $E^{\prime \prime}$, are deduced from these values using eq. (6). 


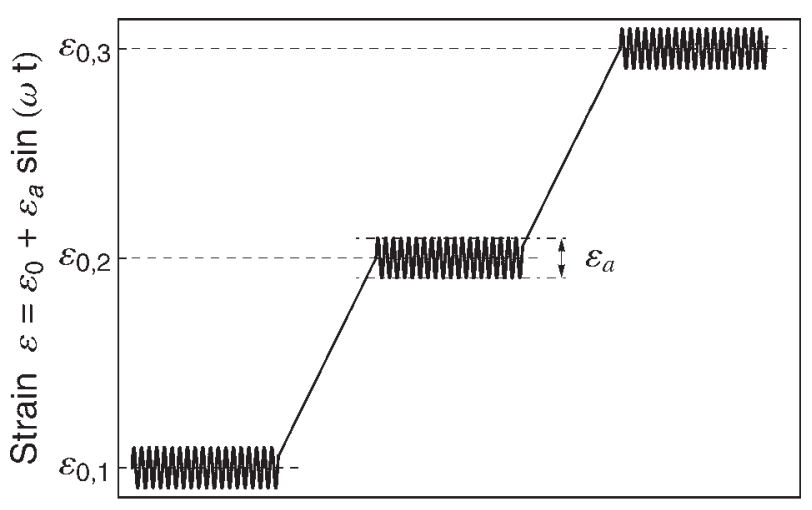

Time $\mathrm{t}$

Figure 2. Schematic representation of the strain history during a PDMA experiment.

$$
\left\|E^{*}\right\|=\sqrt{E^{\prime 2}+E^{\prime \prime 2}}, \quad \tan \delta=\frac{E^{\prime \prime}}{E^{\prime}} .
$$

The storage modulus $E^{\prime}$ quantifies the elastic part of the behavior whereas the loss modulus $E^{\prime \prime}$ corresponds to the quantity of heat dissipated by friction between polymer chains during a strain cycle. $^{24}$

To compare the responses of the propellants, quantifying the measured behavior is necessary. As the curves of the storage and loss moduli according to the logarithm of the prestrain exhibit a linear and a nonlinear domain, each domain is empirically modeled separately. The linear domain is modeled by a line, the nonlinear domain by a power law of the logarithmic prestrain. The threshold between the two domains is optimized as a parameter of the global model. Consequently, a unique model is used to approximate both curves [eq. (7)], where X stands for the storage modulus $E^{\prime}$ or the loss modulus $E^{\prime \prime}$.

$$
X=\left\{\begin{array}{cc}
\alpha+\beta \log \left(\varepsilon_{0}\right) & \text { if } \varepsilon_{0}<\varepsilon_{t}, \\
\alpha+\beta \log \left(\varepsilon_{0}\right)+\gamma\left[\log \left(\frac{\varepsilon_{0}}{\varepsilon_{t}}\right)\right]^{\zeta} & \text { else. }
\end{array}\right.
$$

The constants $\alpha, \beta, \gamma, \varepsilon_{t}$ and $\zeta$ are found using a least-squares optimization algorithm in Mathematica ${ }^{\circledR}$. More precisely, $\alpha$ and $\beta$ are first determined from at least four measurements at low prestrain. The optimized values are then introduced in the second case of eq. (7) to optimize $\varepsilon_{t}, \gamma$, and $\zeta$. Confidence intervals at a $95 \%$ level are determined numerically for each parameter in Mathematica ${ }^{\circledR}$.

Three values are chosen to quantify the behavior. The plateau value $A$ is the model response in the linear domain, at the chosen value $\varepsilon_{0}=\varepsilon_{p}$, see [eq. (8)]. The nonlinearity threshold is directly given by the identified constant $\varepsilon_{t}$. The nonlinearity slope $B$ characterizes the nonlinear domain and is determined from the constants $\varepsilon_{t}, \beta$, $\gamma$, and $\zeta$ according to eq. (9).

$$
\begin{gathered}
A=\alpha+\beta \log \left(\varepsilon_{p}\right), \\
B=\beta+\frac{1}{N} \sum_{i=1}^{N} \gamma \zeta\left[\log \left(\frac{\varepsilon_{t}+\varepsilon_{i}}{\varepsilon_{t}}\right)\right]^{\zeta-1},
\end{gathered}
$$

where $\varepsilon_{p}=0.01 \%, \varepsilon_{i} \in[3 \%, 6 \%]$ and $N=16$.
In the following sections, superscripts $s$ and $l$ are added to the parameters of the model to denote the application for the storage and loss moduli, respectively. Confidence intervals at a $95 \%$ level for $A$ and $B$ are calculated from the ones of the model constants $\alpha, \beta, \varepsilon_{t}, \gamma$, and $\zeta$.

\section{RESULTS AND DISCUSSION}

\section{Swelling Experiments}

Table I lists the filler volume fraction, the chemical nature of molecules in the sol fraction as well as the volume fractions of sol fraction and plasticizer in the binder. These last two quantities are given in volume percentage of the binder to neglect the influence of the filler content.

One should keep in mind that all specimens contain the same amount of fillers because no particle has been added or extracted. However, as the sol fraction substituted evolves from 0 to about $57 \%$ of the reference volume with fillers, the effective filler volume fraction in the resulting composition shows strong variations, from 56.9 to $92.5 \%$.

The specimen DP is the result of the immersion of specimen $\mathrm{E}$ into a 70/30 HTPB/plasticizer mixture. The quantity of sol fraction absorbed in the specimen DP is remarkably lower than the quantity of sol fraction absorbed in specimen D. This could result from the plasticizer depletion of the blend in which specimen DP was immersed. The interaction between HTPB andplasticizer molecules in the bath surrounding the specimen may also prevent the latter from penetrating easily into the network. Finally, because this specimen has been immersed in a mixture of molecules, the ratio of each constituent in the substituted sol fraction is unknown. However, the DSC analysis, performed in the next section, will clarify this uncertainty.

The degree of swelling resulting from this preparation procedure depends on the quantity of sol fraction re-introduced in the specimen (Figure 3 and Table I), which again depends on the ability of the molecules to penetrate the network. In most cases, the final quantity of re-introduced sol fraction is inferior to the extracted one. As a consequence, the macroscopic swelling is

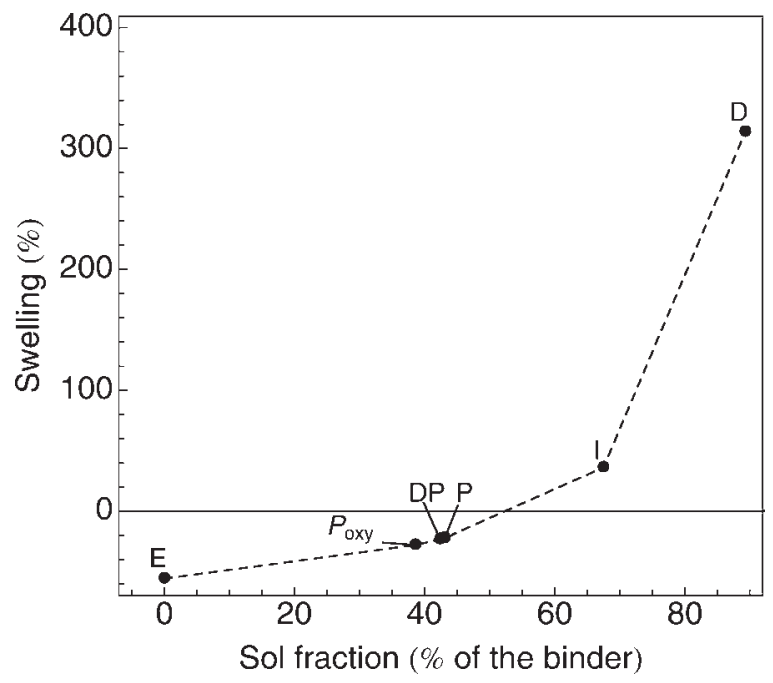

Figure 3. Swelling ratio as a function of sol fraction for all specimens. 


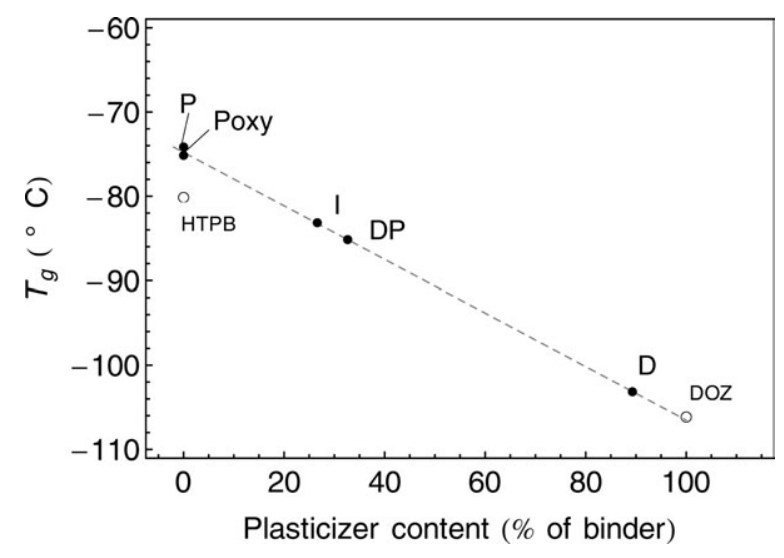

Figure 4. Influence of plasticizer content on the glass transition temperatures.

negative for most specimens. This negative swelling results in a hydrostatic contraction of the network. On the opposite side, the specimen D is clearly in a positive swollen state compared with the reference one and the chains constitutive of its network are extended.

\section{Glass Transition}

The glass transition temperature, $T_{g}$, for each tested specimen is given in Table $I$ and is compared with the $T_{g}$ temperatures of the pure HTPB prepolymer and DOZ plasticizer, measured as $-80.4^{\circ} \mathrm{C}$ and $-106.0^{\circ} \mathrm{C}$ respectively. Because the filler fraction of specimen $\mathrm{E}$ is $92.5 \%$, it contains only $7.5 \%$ of binder and the change in specific heat is not measurable with the DSC apparatus used. As a consequence, this specimen is not included in the following discussion.

It is well known that the glass transition temperature $T_{g}$ depends on the plasticizer content. ${ }^{25}$ This fact is verified in Figure 4 where the measured $T_{g}$ is shown to decrease linearly as the plasticizer content increases. Moreover, this linear dependency may be used to determine the composition of the specimen DP. As it was previously mentioned, this specimen has been immersed in a blend of HTPB and DOZ molecules and the re-introduced sol fraction constitutes $42.4 \%$ of the binder (Table I). However, the final composition of the sol fraction is unknown. Given the glass transition temperature of the specimen DP compared with the linear regression, the volume fraction of plasticizer in this specimen is $32.6 \%$ of the binder. Its sol fraction is, as expected, composed of plasticizer and soluble polymer molecules.

In contrast, $\Delta C_{p}$ is found to depend on both sol fraction and plasticizer content. Therefore and to compare both results, we present in Figure 5 the relative variation of the normalized $\Delta C_{p}$ according to the plasticizer content or the sol-HTPB content. The relative variation of the normalized $\Delta C_{p}$ is $\left(\Delta C_{p}-\right.$ $\left.\Delta C_{p \min }\right) /\left(\Delta C_{p \max }-\Delta C_{p \min }\right)$, where $\Delta C_{p}$ is the actual $\Delta C_{p}$ of the specimen; $\Delta C_{p \max }$ is $0.52 \mathrm{~J} . \mathrm{g}^{-1} \cdot{ }^{\circ} \mathrm{C}^{-1}$ for the sol-HTPB and $0.71 \mathrm{~J} . \mathrm{g}^{-1} \cdot{ }^{\circ} \mathrm{C}^{-1}$ for the DOZ molecules; $\Delta C_{p \min }$ for the solHTPB case was considered as equal to the value $0.21 \mathrm{J.g}^{-1} .{ }^{\circ} \mathrm{C}^{-1}$ of specimen DP; and $\Delta C_{p \text { min }}$ for the plasticizer case as equal to $0.27 \mathrm{~J} . \mathrm{g}^{-1} \cdot{ }^{\circ} \mathrm{C}^{-1}$, i.e., the mean value around which the results of the specimens with low plasticizer contents are scattering.

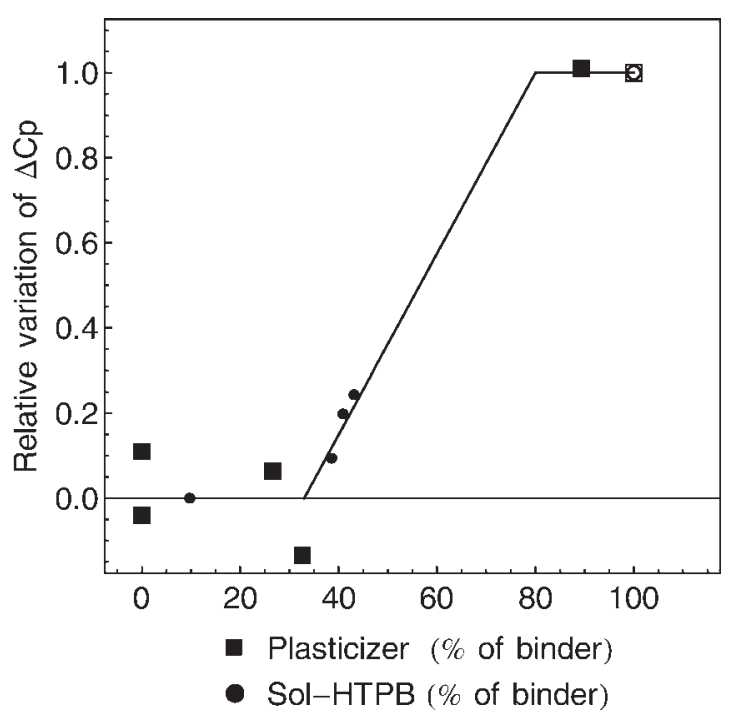

Figure 5. Relative variation of the normalized specific heat change $\Delta C_{p}$ ( $/ \mathrm{g}$ of binder $/{ }^{\circ} \mathrm{C}$ ) according to plasticizer and sol-HTPB content.

Although the number of experimental points is limited, the variations of $\Delta C_{p}$ according to plasticizer content or sol-HTPB content seem to exhibit the same behavior. Two plateaus are suggested on both extreme of the content scale. When a limited quantity $(<30 \%)$ of free molecules is introduced, the normalized $\Delta C_{p}$ seems to remain constant within experimental errors. On the other extreme $(>80 \%)$, the $\Delta C_{p}$ of the binder is close to the one of the free molecules. As a conclusion, the values of the specific heat change are meanly controlled by the largest fraction of the binder, either polymer network or sol fraction. A transition zone is assumed between $30 \%$ and $80 \%$.

\section{PDMA}

Figures 6 and 7 represent the storage and loss moduli obtained from PDMA measurements. All specimens are designed from

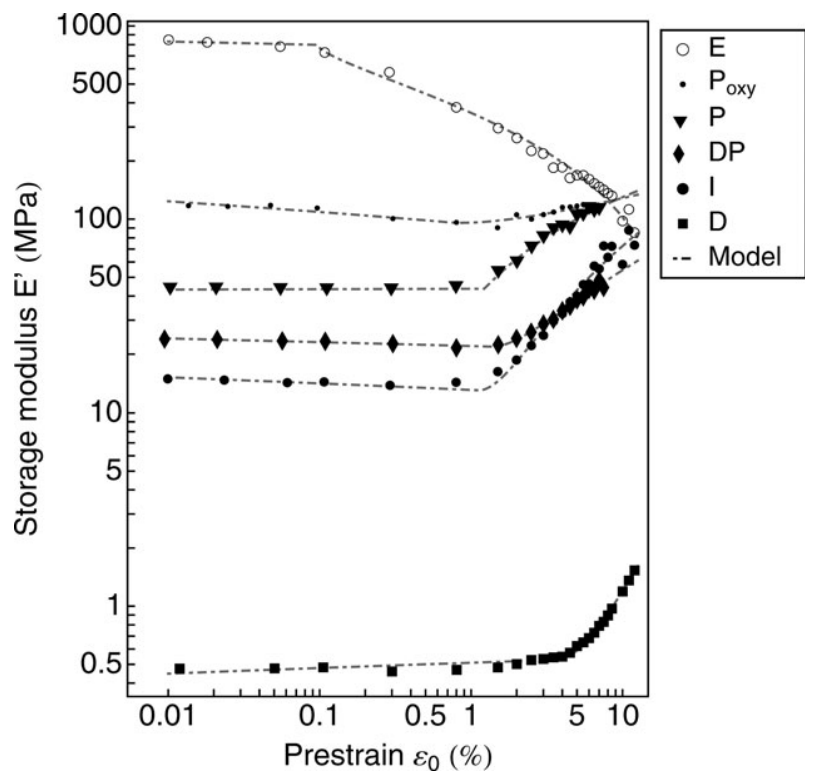

Figure 6. Storage modulus $E^{\prime}$ from PDMA test at room temperature, frequency of $5 \mathrm{~Hz}$ and $\varepsilon_{a}=0.01 \%$. 


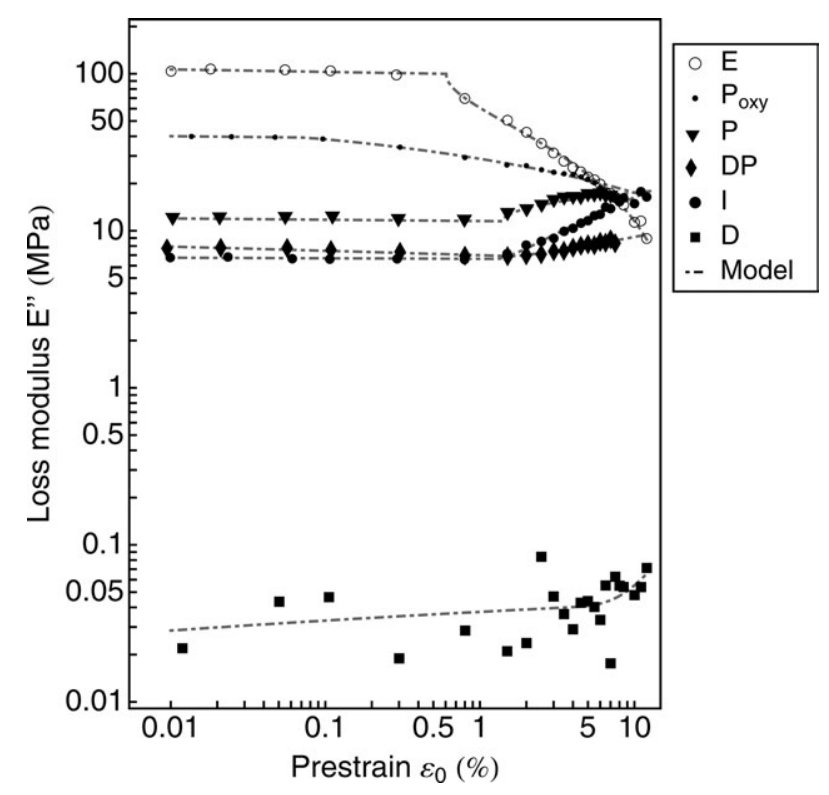

Figure 7. Loss modulus $E^{\prime \prime}$ from PDMA test at room temperature, frequency of $5 \mathrm{~Hz}$ and $\varepsilon_{a}=0.01 \%$.

the same original propellant composition (specimen I). Therefore, they have an identical polymer network and differ only in their sol fraction nature and quantity. However, this difference has a significant influence on the measured mechanical behaviors, as showed in Figures 6 and 7. The mathematical model, eq. (7), is fitted for each result. The plateau values $A$, thresholds $\varepsilon_{t}$, and nonlinearity slopes $B$ are discussed according to the specimen compositions. Finally, the ability of the model to correctly represent such different behaviors is discussed in the last section.

Material D presents a highly scattered loss factor measurement (Figure 7). Because the strain amplitude $\varepsilon_{a}$ is not high enough, loss factor values are too low to enable reliable measurements. At a single strain amplitude of $0.1 \%$, that is 10 times larger, the measurement gets less scattered. Nevertheless, because specimen E could not bear without break a larger strain amplitude, tests are still performed at $\varepsilon_{a}=0.01 \%$ for the sake of comparison.

Linear Domain. Parameters $A^{\mathrm{s}}$ and $A^{\mathrm{l}}$ characterize the linear domain, as the prestrain $\varepsilon_{p}$ is lower than $\varepsilon_{t}$, see [eq. (8)]. Plateau values $A^{\mathrm{s}}$ and $A^{1}$ decrease as the swelling increases (Figure 8 ). The fact that a specimen is either under extension or contraction mode has apparently a minor influence on the linear behavior, because the plateau values seem to decrease in a nearly monotonic way, from $1000 \mathrm{MPa}$ to less than $0.05 \mathrm{MPa}$, as the degree of swelling increases. However, the dependency of the modulus on the swelling degree is remarkably stronger in the contraction zone than in the extension one.

The decrease in network moduli on swelling may be ascribed to several origins. First, swelling leads to a decrease in the density of polymer network per unit volume which in turn leads to a decrease in intermolecular constraints. Second, this diminution has been observed previously and attributed to the increase in the free volume of the sample leading to a decrease in intramo- lecular constraints. ${ }^{10}$ Third, swelling also decreases the effective filler fraction, ${ }^{11}$ which ends up inevitably decreasing $A^{\mathrm{s}}$ and $A^{1}{ }^{11,24}$

Furthermore, we may notice that the nature of the sol fraction does not seem to be a driving force behind the decrease in moduli, observed in Figure 8. One possible cause is that most of the specimens presented in the contraction zone of this figure do not contain any plasticizer (only sol-HTPB fraction) whereas specimens at higher swelling ratio are swollen exclusively by plasticizer molecules. We may mention here that, when DMA experiments have been performed on filled rubber swollen in various liquids, the viscosity of the liquids had similarly no influence on the observed viscoelastic behavior. ${ }^{11}$

Both the filler fraction and the state of deformation of the elementary chain in the network (swelling determined in reference to its unperturbed state) have a predominant influence on the behavior. In this perspective, it is expected that the hydrostatic contraction, caused by the negative swelling, leads to a stronger dependency of modulus on deformation than the one observed in the extension mode.

Nonlinear Domain. The nonlinearity thresholds $\varepsilon_{t}^{s}$ and $\varepsilon_{t}^{l}$ define the prestrain at which nonlinear mechanisms are initiated. Thresholds $\varepsilon_{t}$ appear to increase with the degree of swelling, with an apparent plateau around the unperturbed zero swelling (Figure 9). The nonlinearity thresholds of the storage and loss moduli according to the imposed prestrain are generally attributed to the finite extensibility of the network. ${ }^{11,19,20}$ Moreover, the finite extensibility of a rubber network decreases if the network is swollen (given that uniaxial tension is added to the isotropic deformation imposed by swelling). ${ }^{10,26}$ Because the opposite evolution is observed in Figure 9, the determined thresholds can not result uniquely from the finite extensibility

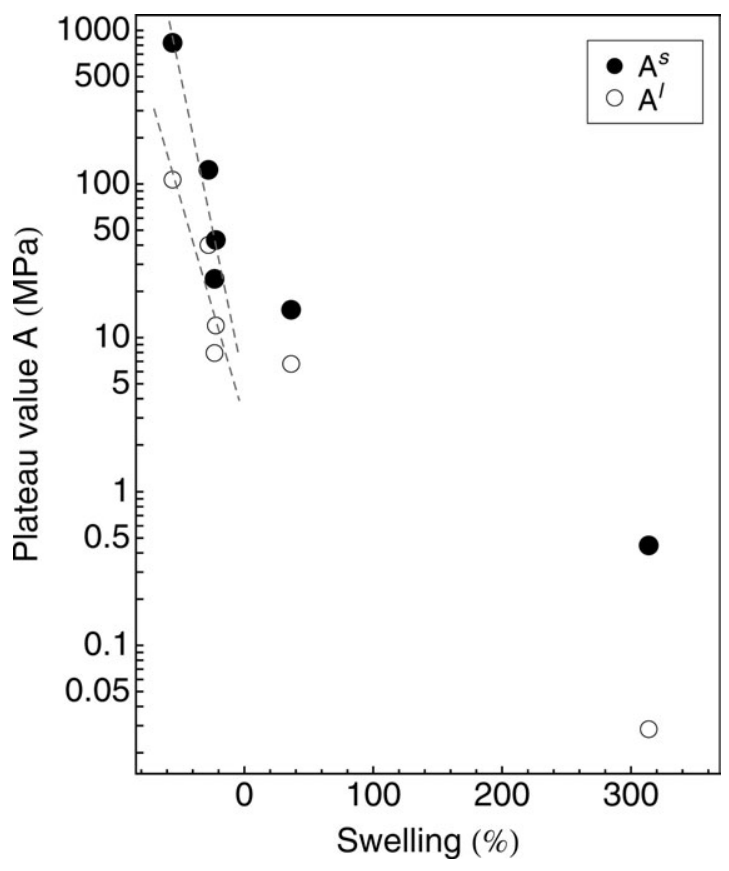

Figure 8. Plateau values $A^{\mathrm{s}}$ and $A^{1}$ according to swelling degree. 


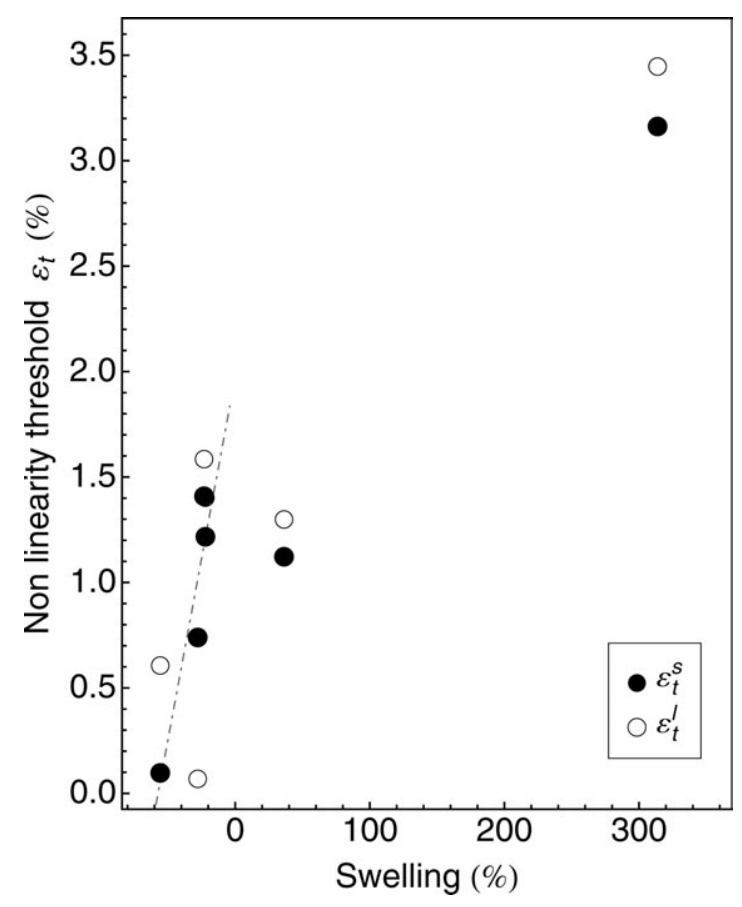

Figure 9. Nonlinearity thresholds $\varepsilon_{t}^{s}$ and $\varepsilon_{t}^{l}$ according to swelling degree.

of the polymer network. However, as pointed out in the next section, the lack of precision in the threshold fit prevents their full interpretation. Consequently, $\varepsilon_{t}^{s}$ and $\varepsilon_{t}^{l}$ will not be discussed any further.

The most significant effect remains that as long as the specimen is contracted, nonlinearity slopes $B^{\mathrm{s}}$ and $B^{\mathrm{l}}$ increase as the swelling increases (Figure 10). Slopes $B^{\mathrm{s}}$ and $B^{\mathrm{l}}$ increase from negative to positive values in a strikingly linear way, as long as the swelling remains negative. One can also notice that the slope is null for a swelling different from zero and the nonlinearity limit is somewhere between -23 and $-55 \%$ of swelling. In addition, a change in the behavior is observed as the swelling approaches its zero value. When the swelling is positive, nonlinearity slopes decrease as the swelling increases.

These results highlight the role of the polymer network, even though the material is highly filled. They suggest that the pertinent factor behind this behavior is the swelling of the polymer network phase. Indeed, in these filled rubber composites, the polymer matrix is the only phase that is able to support the sol fraction, plasticizer and/or unlinked polymer. It is thus the only conceivable origin of the delimitation of the material behavior into two distinct zones: the contraction and extension zones.

In the contraction zone, the most compressed specimen, $\mathrm{E}$, is near its state of maximum compressibility. The hydrostatically compressed polymer phase is highly restricted, resulting in reduced chain mobility and increased storage modulus of up to about $1000 \mathrm{MPa}$ as shown in Figure 8. The applied uniaxial prestrain leads to a storage modulus nonlinearity at very low values of the prestrain. Hence, some of the polymer restriction is released by the applied prestrain and the moduli are reduced with high negative nonlinearity slopes $B^{\mathrm{s}}$ and $B^{\mathrm{l}}$ (Figure 10).
On swelling, the polymer hydrostatic restriction is progressively released and the moduli decrease rather strongly, as seen in Figure 8 for specimens $\mathrm{P}_{\text {oxy }}$ DP, and P. Consequently, the effect of the swelling is progressively reduced compared with the effect observed on specimen E. The system behavior approaches gradually the behavior of the unperturbed material, i.e., swelling equal to 0 .

Although the loading here is different from a simpler strain amplitude scanning, this interpretation, using the release of internal constraints, can be compared with the mechanisms associated with the Payne effect in filled elastomers. ${ }^{27}$ The Payne effect is the decrease in the storage and loss moduli resulting from an increase in the strain amplitude. It has been suggested that, as the strain amplitude increases, the carbon black filler network is destroyed, internal constraints are released, and the moduli are lowered. ${ }^{24,28}$ However, because the nature, quantity, and size of the fillers are different in the case of highly filled elastomers, this interpretation is controversial. ${ }^{29}$ In this study, we are tempted to attribute the internal constraints not to an initial filler network but to the hydrostatic contraction of the whole specimen.

Moreover, as illustrated in Figure 10, the nonlinearity slopes, $B$, increase strongly at low swelling degrees and go from negative to positive values on swelling. It is noticeable that a partial release of the polymer restrictions by a slight degree of swelling increases the modulus with increasing prestrain. This increase in modulus with prestrain has been observed on filled elastomers at higher prestrains and has been attributed to the finite extensibility of the network. ${ }^{11,16,19,20}$ However, because all the specimens have the same network here, the influence of the swelling on the whole system has to be taken into account. We suggest that the observed mechanical behavior is related to some uniaxial alignments of the solid filler particles. It has been shown that, in highly filled systems like solid propellants, stress concentrations appear between adjacent close particles depending

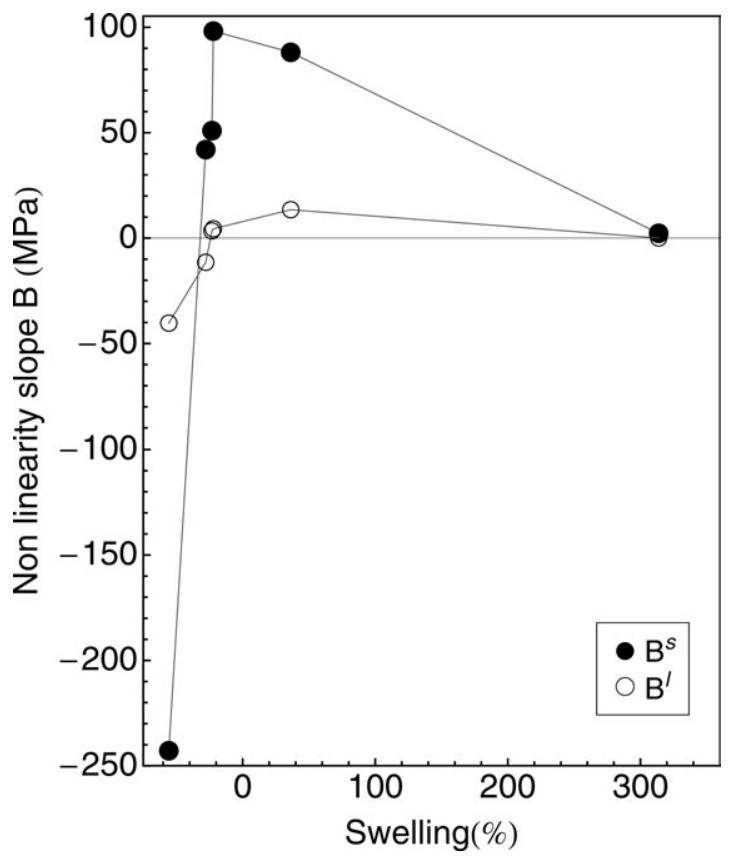

Figure 10. Nonlinearity slopes $B^{\mathrm{s}}$ and $B^{\mathrm{l}}$ according to swelling degree. 
on their size. ${ }^{8}$ Moreover, bands of increased stress are created as those particles align in the direction of the applied strain. The binder between those particles is highly strained and reaches its finite extensibility at lower prestrain. Finally, as long as the system is in the compressed state, the probability of particle aligning is increased by the release of the polymer restriction.

Within the limits of the hypothetical unperturbed state (swelling $=0 \%$-though none of our specimens corresponds to this discriminating state-the moduli values result from the filler loading and the degree of cross-linking of the polymer; the thresholds $\varepsilon_{t}$ may remain constant at a plateau value but results in Figure 9 are not accurate enough to confirm such a hypothesis. Because chains are free of restriction in the unperturbed state, the probability of particles aligning is the highest and the rates of moduli increase, $B^{\mathrm{s}}$ and $B^{\mathrm{l}}$, should be at their upper level (Figure 10).

In the extension zone, the moduli keep decreasing with swelling as expected (Figure 8). Similarly to the other highly filled specimens, the nonlinearity manifests itself by an increase in the moduli with prestrain, because of particle aligning. However, the nonlinearity slopes, $B^{\mathrm{s}}$ and $B^{\mathrm{l}}$, decrease with swelling (Figure 10). The system behaves as if the plasticizer molecules dilute the particles and prevent their alignment.

Limits of the Proposed Model. Confidence intervals at a $95 \%$ level have been calculated for each parameter $A, \varepsilon_{t}$, and $B$. Comparing with the optimized values, a relative error is determined. The error for each parameter is usually between 10 and $50 \%$. The errors on the parameters are quite large, despite the good agreement of the total model with experimental measurements, as shown on Figures 11 and 12 for the specimen DP.

The model could be too simple to represent the variety of behaviors observed here. For numerous cases, the error is higher for $\varepsilon_{t}$ and reaches extremely high values beyond $100 \%$. Threshold determination constitutes the weak point of this model.

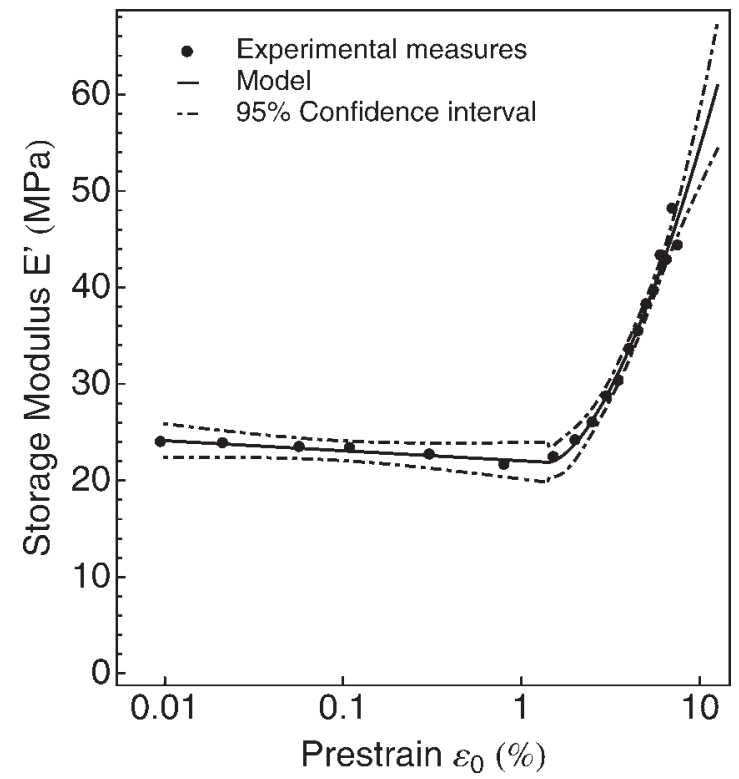

Figure 11. Storage modulus $E^{\prime}$ for specimen DP at room temperature, frequency of $5 \mathrm{~Hz}$ and $\varepsilon_{a}=0.01 \%$.

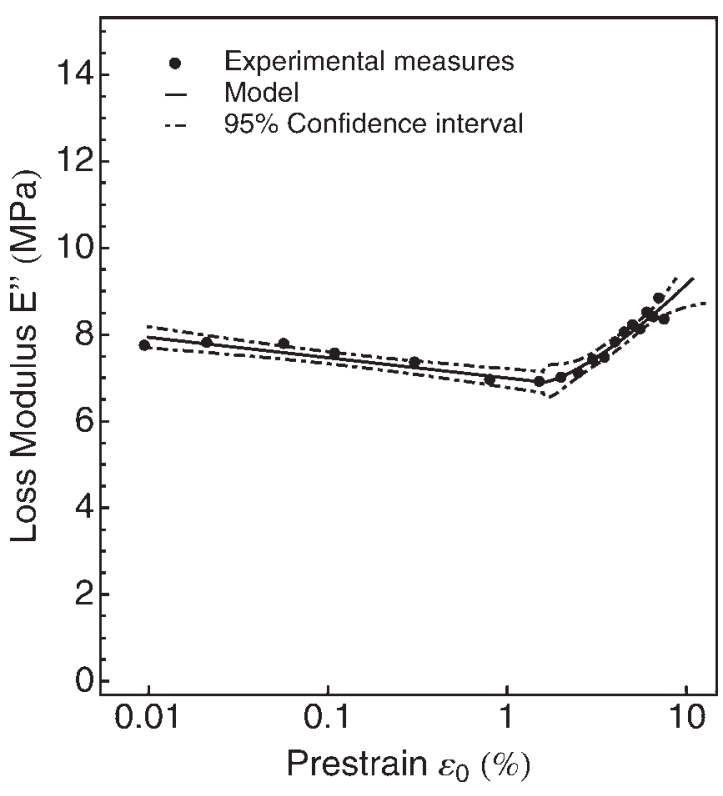

Figure 12. Loss modulus $E^{\prime \prime}$ for specimen DP at room temperature, frequency of $5 \mathrm{~Hz}$ and $\varepsilon_{a}=0.01 \%$.

\section{CONCLUSION}

The aim of this study is to specify the influence of the sol fraction on the viscoelastic properties of a solid propellant. Designed specimens are prepared by introducing variable quantities of sol fraction into the reference propellant. The quantity of sol fraction directly determines the swelling degree applied to the specimen. The advantage of this procedure is that the specimens are all prepared from the same propellant composition. The filler quantity and the polymer network are identical whereas the sol fraction varies in chemical nature and quantity. A correspondence between the swelling degree and the state of contraction or extension of the polymer network is established. In addition, as a consequence of the swelling, the effective filler fraction is strongly modified.

Prestrained DMA experiments are performed on each specimen. A mathematical function is suggested to model the nonlinear behavior. Results show that the nature of the sol fraction is not the predominant factor. In the linear domain, the state of contraction/extension of the polymer network and the effective filler fraction, both consequences of the swelling, control the values of the storage and loss moduli. Similarly, the analysis of the nonlinearity highlights the predominant role played by the swelling of the polymer network phase. Moreover, a local mechanism involving particles aligning in the direction of the applied prestrain may modify the stress applied locally to the polymer network and lead to the observed moduli increases. This theory explains the observed nonlinear behavior and the influence of the swelling on the viscoelastic properties.

\section{ACKNOWLEDGMENTS}

The authors thank Mrs Amiet (DGA) for supporting this project and M. Grévin, N. Quaglia, and P. Pinard for their contribution to the experimental work. The work of A.A. is financially supported by the Délégation Généale pour l'Armement (DGA), France. 


\section{REFERENCES}

1. Ozupek, S.; Becker, E. J. Eng. Mater. Technol.-Trans. ASME 1992, 114, 111.

2. Ozupek, S.; Becker, E. J. Eng. Mater. Technol.-Trans. ASME 1997, 119, 125

3. Ravichandran, G.; Liu, C. Int. J. Solid Struct. 1995, 32, 979.

4. Nadot-Martin, C.; Trumel, H.; Dragon, A. Eur. J. Mech. A: Solid 2003, 22, 89.

5. Nadot-Martin, C.; Dragon, A.; Trumel, H.; Fanget, A. J. Theor. Appl. Mech. 2006, 44, 553.

6. Nadot-Martin, C., Touboul, M., Dragon, A. and Fanget, A. (2010) Direct Scale Transition Approach for Highly-filled Viscohyperelastic Particulate Composites: Computational Study, in Multiscale Modeling of Heterogenous Materials: From Microstructure to Macro-scale Properties (ed O. Cazacu), ISTE, London, UK.

7. Xu, F.; Aravas, N.; Sofronis, P. J. Mech. Phys. Solids 2008, 56,2050

8. Matous, K.; Inglis, H.; Gu, X.; Rypl, D.; Jackson, T.; Geubelle, P. Compos. Sci. Technol. 2007, 67, 1694.

9. Fujita, H. Macromolecules 1993, 26, 643.

10. Davies, C.; Thomas, A.; Akutagawa, K. Progr. Rubber Plast. Technol. 1996, 12, 174.

11. Busfield, J.; Deeprasertkul, C.; Thomas, A. Polymer 2000, 41, 9219.

12. Azoug, A. Ph.D. Thesis, Ecole Polytechnique, 2010.

13. Mason, P. J. Appl. Polym. Sci. 1959, 1, 63.
14. Suphadon, N.; Thomas, A.; Busfield, J. J. Appl. Polym. Sci. 2009, 113, 693 .

15. Suphadon, N.; Thomas, A.; Busfield, J. J. Appl. Polym. Sci. 2010, 117, 1290.

16. Meinecke, E.; Maksin, S. Rubber Chem. Technol. 1981, 54, 857.

17. Sullivan, J.; Demery, V. J. Polym. Sci. Polym. Phys. Ed. 1982, 20, 2083.

18. Arai, K.; Ferry, J. Rubber Chem. Technol. 1986, 59, 605.

19. Voet, A.; Morawski, J. Rubber Chem. Technol. 1974, 47, 765.

20. Dutta, N.; Tripathy, D. Polym. Test. 1990, 9, 3.

21. Warley, R.; Feke, D.; Manas-Zloczower, I. J. Appl. Polym. Sci. 2007, 104, 2197.

22. Adicoff, A.; Lepie, A. J. Appl. Polym. Sci. 1970, 14, 953.

23. Desgardin, N.; Chevalier, S.; Grevin, M. Duree de vie, Technical Report CRB N¹2/06/CRB/DPS/CRA/DR 2006.

24. Medalia, A. Rubber Chem. Technol. 1978, 51, 437.

25. Van Krevelen, D.; Hoftyzer, P. Properties of Polymers: Their Estimation and Correlation with Chemical Structure, 2nd ed.; Amsterdam; New York: Elsevier Scientific Pub. Co. 1976.

26. Mullins, L. J. Appl. Polym. Sci. 1959, 2, 257.

27. Payne, A.; Whittaker, R. Rubber Chem. Technol. 1971, 44, 440.

28. Kraus, G. J Appl Polym Sci: Applied Polymer Symposium 1984, 39, 75

29. Stacer, R.; Hubner, C.; Husband, D. Rubber Chem. Technol. 1990, 63, 488 . 